\title{
Observation of quantum droplets in a heteronuclear bosonic mixture
}

\author{
C. D’Errico $\odot,{ }^{1,2, *}$ A. Burchianti $\odot,{ }^{1,2, \dagger}$ M. Prevedelli $\odot,{ }^{3}$ L. Salasnich $\odot,{ }^{1,4}$ F. Ancilotto $\odot,{ }^{4,5}$ \\ M. Modugno ${ }^{6,7}$ F. Minardi $\odot,{ }^{1,2,3}$ and C. Fort ${ }^{1,2}$ \\ ${ }^{1}$ CNR, Istituto Nazionale di Ottica, 50019 Sesto Fiorentino, Italy \\ ${ }^{2}$ LENS and Dipartimento di Fisica e Astronomia, Università di Firenze, 50019 Sesto Fiorentino, Italy \\ ${ }^{3}$ Dipartimento di Fisica e Astronomia, Università di Bologna, 40127 Bologna, Italy \\ ${ }^{4}$ Dipartimento di Fisica e Astronomia "Galileo Galilei" and CNISM, Università di Padova, 35131 Padova, Italy \\ ${ }^{5}$ CNR-IOM Democritos, 265-34136 Trieste, Italy \\ ${ }^{6}$ Departamento de Fisíca Teórica e Historia de la Ciencia, Universidad del Pais Vasco UPV/EHU, 48080 Bilbao, Spain \\ ${ }^{7}$ IKERBASQUE, Basque Foundation for Science, 48013 Bilbao, Spain
}

(Received 10 July 2019; published 6 December 2019)

\begin{abstract}
We report on the formation of heteronuclear quantum droplets in an attractive bosonic mixture of ${ }^{41} \mathrm{~K}$ and ${ }^{87} \mathrm{Rb}$. We observe long-lived self-bound states, both in free space and in an optical waveguide. In the latter case, the dynamics under the effect of a species-dependent force confirms their bound nature. By tuning the interactions from the weakly to the strongly attractive regime, we study the transition from expanding to localized states, in both geometries. We compare the experimental results with beyond-mean-field theory and we find good agreement in the full range of explored interactions. Our findings provide access to the production of long-lived droplets with important implications for further research.
\end{abstract}

DOI: 10.1103/PhysRevResearch.1.033155

\section{INTRODUCTION}

Interactions are ubiquitous in nature and understanding their effects is a primary challenge in physics, especially in many-body quantum systems. Generally, mean-field (MF) theories reproduce well a plethora of phenomena related to interparticle interactions. However, this approach fails when quantum fluctuations are non-negligible. The first-order correction to the MF energy, the so-called Lee-Huang-Yang (LHY) term, was first calculated [1] to describe bosons with hard-core repulsion. In ultracold gases the LHY term is normally negligible and only recently it has been experimentally investigated [2-6]. Nevertheless, there are situations where the LHY and MF contributions can be of the same order. For instance, in attractive bosonic mixtures the repulsive LHY term may stabilize the system against collapse, leading to the formation of self-bound droplets. Although very dilute, these states have a liquidlike behavior, characterized by a core with uniform density in the large atom number limit [7].

While originally predicted for Bose-Bose mixtures, quantum droplets were first realized in dipolar gases, by exploiting the competition between contact repulsion and anisotropic dipole-dipole attraction [8-13]. Recently, tuning contact interactions through a Feshbach resonance, quantum droplets have been observed also in a spin mixture of ${ }^{39} \mathrm{~K}$, both in

\footnotetext{
*derrico@lens.unifi.it

†burchianti@lens.unifi.it

Published by the American Physical Society under the terms of the Creative Commons Attribution 4.0 International license. Further distribution of this work must maintain attribution to the author(s) and the published article's title, journal citation, and DOI.
}

the presence of an external potential [14,15] and in free space [16]. By studying collisions between droplets it has been possible to observe the crossover between compressible and incompressible regimes [17]. Free space ${ }^{39} \mathrm{~K}$ droplets have a short lifetime (only few milliseconds), limited by threebody losses [16,17], motivating the quest for stable long-lived droplets in different atomic mixtures. Longer lifetimes could indeed allow the investigation of many peculiar features of these states, such as the characterization of the incompressible regime and the observation of self-evaporation [7].

In this work we study the formation of quantum droplets in a heteronuclear Bose-Bose mixture of ${ }^{41} \mathrm{~K}$ and ${ }^{87} \mathrm{Rb}$ where the two species experience different trapping potentials. We exploit Feshbach resonances for tuning interspecies interactions. In particular, we explore the transition from the weakly to the strongly attractive regime, beyond the MF threshold for collapse, where self-bound quantum droplets are predicted to exist. We observe that in the absence of any external trapping potential, the strongly attractive mixture remains localized on a timescale of several tens of milliseconds, consistently with the formation of long-lived self-bound droplets. Furthermore, we study the size evolution and the center-of-mass dynamics of the mixture in a horizontal optical waveguide, in the presence of a species-dependent magnetic force. Also in this geometry, where both the LHY correction and the radial confinement provide a mechanism to prevent the collapse [18], we observe the formation of a localized bound state whose centerof-mass follows a trajectory intermediate between those of two weakly attractive ${ }^{41} \mathrm{~K}$ and ${ }^{87} \mathrm{Rb}$ clouds. By studying the dynamics, we extract the number ratio of ${ }^{41} \mathrm{~K}$ and ${ }^{87} \mathrm{Rb}$ atoms forming the bound state, and we find it consistent with the value predicted by the theory [7]. Our experimental findings are reproduced well by numerical simulations performed by 
using two coupled generalized Gross-Pitaevskii (GP) equations $[19,20]$, including the LHY correction for heteronuclear mixtures $[7,21]$.

\section{EXPERIMENT}

We produce a binary condensate of ${ }^{41} \mathrm{~K}$ and ${ }^{87} \mathrm{Rb}$ both prepared in the $\left|F=2, m_{F}=2\right\rangle$ state in a crossed optical dipole trap [22]. To tune the interspecies interaction, two microwave pulses transfer both species in the $\left|F=1, m_{F}=1\right\rangle$ state and a vertical homogeneous magnetic field is increased to $\simeq 71 \mathrm{G}$, lying between two Feshbach resonances [23]. Due to their different masses, ${ }^{41} \mathrm{~K}$ and ${ }^{87} \mathrm{Rb}$ atoms have a differential vertical gravitational sag and their equilibrium positions are separated by $\sim 15 \mu \mathrm{m}$ in the optical trap. We compensate for this offset by compressing the dipole trap and by adding a vertical magnetic field gradient $b_{z}=-16 \mathrm{G} / \mathrm{cm}[24,25]$, exploiting the different magnetic moments of the two species $\left(\mu_{\mathrm{K}}=-0.83 \mu_{B}\right.$ and $\mu_{\mathrm{Rb}}=-0.52 \mu_{B}$, at $\left.70 \mathrm{G}\right)$, as shown in Appendix A. At this stage, we normally have a binary condensate with $N_{\mathrm{K}} \simeq(1-2) \times 10^{4}$ and $N_{\mathrm{Rb}} \simeq(4-6) \times 10^{4}$ atoms, confined in an approximately harmonic potential with different frequencies for the two atomic species: $\left(v_{x}, v_{y}, v_{z}\right) \simeq$ $(130,90,180) \mathrm{Hz}$ for ${ }^{41} \mathrm{~K}$ and $(100,70,130) \mathrm{Hz}$ for ${ }^{87} \mathrm{Rb}$. The homonuclear and heteronuclear scattering lengths are, respectively, $a_{\mathrm{K}}=65 a_{0}$ [26] and $a_{\mathrm{Rb}}=100.4 a_{0}$ [27], and $a_{\mathrm{KRb}} \simeq 0 a_{0}[28,29]$. To enter the attractive regime, we then change the magnetic field to the desired value $B_{F}$ in $30 \mathrm{~ms}$, thus tuning $a_{\mathrm{KRb}}$, as detailed in Appendix A. The mixture is completely characterized in terms of the intraspecies $g_{\mathrm{K}}=4 \pi \hbar^{2} a_{\mathrm{K}} / m_{\mathrm{K}}$ and $g_{\mathrm{Rb}}=4 \pi \hbar^{2} a_{\mathrm{Rb}} / m_{\mathrm{Rb}}$ and interspecies $g_{\mathrm{KRb}}=2 \pi \hbar^{2} a_{\mathrm{KRb}} / m_{r}$ coupling constants, with $m_{\mathrm{K}}$ and $m_{\mathrm{Rb}}$ the atomic masses and $m_{r}$ the reduced mass. The onset of the MF collapse regime corresponds to $\delta g=g_{\mathrm{KRb}}+\sqrt{g_{\mathrm{K}} g_{\mathrm{Rb}}}=0$ $\left(a_{\mathrm{KRb}}=-75.4 a_{0}\right)[30]$. We explore the crossover from the weak $\left(a_{\mathrm{KRb}}<0, \delta g>0\right)$ to the strong $(\delta g<0)$ attractive regime. For the measurements in free space, the trap beams and the magnetic field gradient $b_{z}$ are simultaneously switched off and the atoms are imaged in time of flight (TOF). For the expansion in the horizontal waveguide (along the $\hat{y}$ direction) one of the two beams of the dipole trap [labeled as crossed beam in Fig. 1(a)] is slowly turned off by linearly reducing its intensity in $10 \mathrm{~ms}$ and the atoms are imaged in situ after a given evolution time. In the waveguide the atoms experience a radial trapping potential with average frequencies $v_{r} \simeq$ $160 \mathrm{~Hz}(120 \mathrm{~Hz})$ and an axial antitrapping potential, produced by the magnetic field gradient, with $v_{y} \simeq-1.8 \mathrm{~Hz}(-0.6 \mathrm{~Hz})$ for ${ }^{41} \mathrm{~K}\left({ }^{87} \mathrm{Rb}\right)$.

\section{RESULTS}

\section{A. Dynamics in free space}

We first consider the dynamics in free space. In Fig. 1(b) we show typical absorption images of the two condensates in the $x z$ plane and with a TOF of $24.5 \mathrm{~ms}$ for $\delta g>0$ (top) and $\delta g<0$ (bottom). In the former case both ${ }^{41} \mathrm{~K}$ and ${ }^{87} \mathrm{Rb}$ samples freely expand, while in the latter case we observe that a fraction of both species bounds and forms a small and dense component. The different shape of ${ }^{41} \mathrm{~K}$ and ${ }^{87} \mathrm{Rb}$ clouds for $\delta g<0$ is a consequence of our imaging procedure. To detect

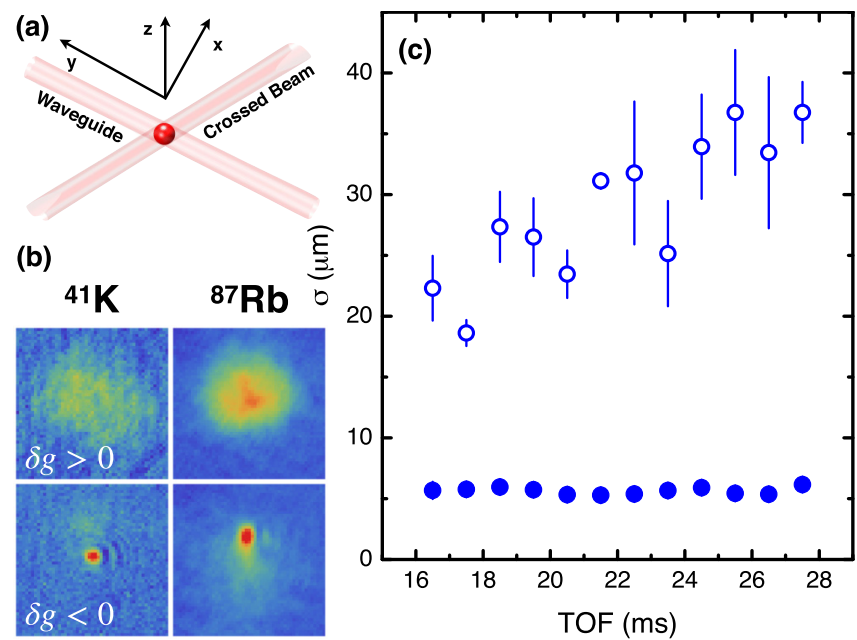

FIG. 1. (a) Schematics of the geometry of the crossed trap. (b) Absorption images in the $x z$ plane $\left(190 \times 190 \mu \mathrm{m}^{2}\right)$ after a TOF of $24.5 \mathrm{~ms}(27 \mathrm{~ms})$ for ${ }^{41} \mathrm{~K}\left({ }^{87} \mathrm{Rb}\right)$ condensate in the weakly attractive (top row) and in the strongly attractive (bottom row) regimes. The ${ }^{41} \mathrm{~K}$ atoms are imaged at the target Feshbach field, while the ${ }^{87} \mathrm{Rb}$ atoms are imaged at $B=0 \mathrm{G}$ after an additional TOF of $2.5 \mathrm{~ms}$. (c) Average size $\sigma=\sqrt{\sigma_{x} \sigma_{z}}$ of the ${ }^{41} \mathrm{~K}$ cloud as a function of the TOF in free space for two values of the interspecies scattering length: $a_{\mathrm{KRb}}=(-17.5 \pm 1.4) a_{0}$ (open circles) and $a_{\mathrm{KRb}}=(-84.5 \pm 1.6) a_{0}$ (closed circles). The error bars correspond to a standard deviation of typically five independent measurements.

both atomic species, in fact, we first take an absorption image of the ${ }^{41} \mathrm{~K}$ sample at the magnetic field $B_{F}$, then we switch off $B_{F}$, and after further $2.5 \mathrm{~ms}$ of TOF we take the image of ${ }^{87} \mathrm{Rb}$ atoms. The actual size of the bound state can be measured only in the first image, after which the state is dissociated and the ${ }^{87} \mathrm{Rb}$ cloud expands. In Fig. 1(c) we plot the average width (rms) $\sigma=\sqrt{\sigma_{x} \sigma_{z}}$ of the ${ }^{41} \mathrm{~K}$ sample as a function of TOF for both interaction regimes. Whereas for $\delta g>0$ (open circles) $\sigma$ increases in time, for $\delta g<0$ (closed circles) it does not exceed our imaging resolution $(5 \mu \mathrm{m})$ up to $28 \mathrm{~ms}$ of TOF, indicating the formation of a droplet state. In our mixture the droplet density is expected to be one order of magnitude smaller than in ${ }^{39} \mathrm{~K}$ at the same $\delta g$, leading to a substantial reduction of the three-body losses. This is consistent with a lifetime two orders of magnitude longer. Such a difference is mainly due to the larger scattering lengths of ${ }^{41} \mathrm{~K}$ and ${ }^{87} \mathrm{Rb}$, since the density scales as $n \sim(\delta g / g)^{2} a^{-3}$ for approximately equal scattering lengths, as discussed in Appendix B. In the experiment, for a TOF greater than $28 \mathrm{~ms}$, we observe that the atomic clouds start to expand. We attribute this to the variation of $a_{\mathrm{KRb}}$, since for a longer TOF the atoms fall off the region where the Feshbach field is spatially homogeneous. Nevertheless, this time is longer than the maximum observation time of ${ }^{39} \mathrm{~K}$ droplets in free space [16]. In order to increase the observation time, one could exploit a magnetic gradient $b_{z}$ to levitate the droplet, with an average magnetic moment per particle

$$
\mu_{\mathrm{KRb}}=\frac{N_{\mathrm{K}}^{d} \mu_{\mathrm{K}}+N_{\mathrm{Rb}}^{d} \mu_{\mathrm{Rb}}}{N_{\mathrm{K}}^{d}+N_{\mathrm{Rb}}^{d}},
$$

where $N_{\mathrm{K}}^{d}$ and $N_{\mathrm{Rb}}^{d}$ are the number of ${ }^{41} \mathrm{~K}$ and ${ }^{87} \mathrm{Rb}$ atoms forming the bound state. However, we observed that, due to 


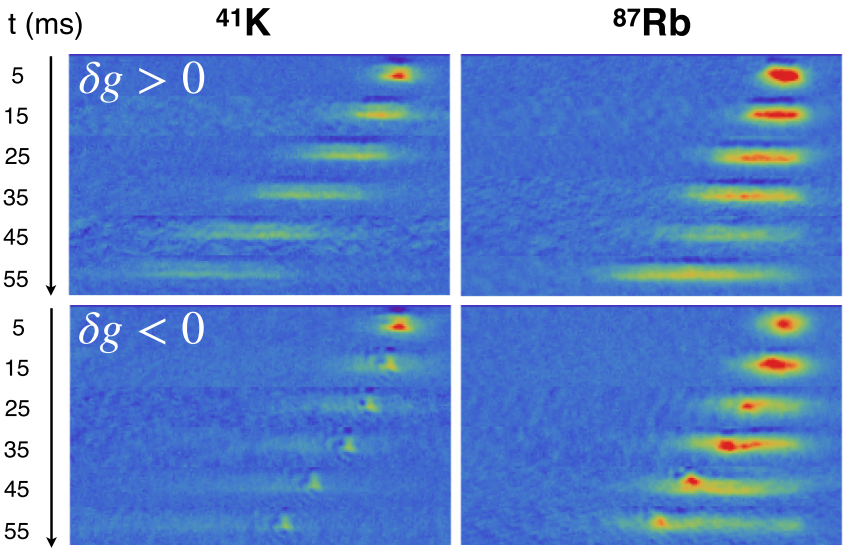

FIG. 2. In situ absorption images in the $y z$ plane of the atoms expanding in a horizontal waveguide $\left(760 \times 266 \mu \mathrm{m}^{2}\right)$ at increasing (from top to bottom) evolution time $t$ in the weakly attractive regime $a_{\mathrm{KRb}}=(-11.4 \pm 1.4) a_{0}$ (top row) and in the strongly attractive one $a_{\mathrm{KRb}}=(-82.4 \pm 1.6) a_{0}$ (bottom row). Both ${ }^{41} \mathrm{~K}$ and ${ }^{87} \mathrm{Rb}$ atoms are imaged as described in Fig. 1.

the species-dependent magnetic force, the droplet breaks up for $b_{z} \gtrsim 5 \mathrm{G} / \mathrm{cm}$. Thus, we apply a lower magnetic field gradient $b_{z}=1.1 \mathrm{G} / \mathrm{cm}$, which, even if too small to compensate for gravity, reduces the variation of the Feshbach field during the TOF. ${ }^{1}$ In addition, it introduces a speciesdependent acceleration sufficient to spatially discriminate the bound and the unbound atoms. In the images at $\delta g<0$ [Fig. 1(b), bottom], we note indeed the presence of a halo surrounding the localized clouds of both ${ }^{41} \mathrm{~K}$ and ${ }^{87} \mathrm{Rb}$. Due to the finite temperature, a fraction of atoms remains unbound. Moreover, for an efficient sympathetic cooling of the mixture, the atom number of ${ }^{87} \mathrm{Rb}$ is typically three or four times larger than that of ${ }^{41} \mathrm{~K}$, leading to an excess of unbound ${ }^{87} \mathrm{Rb}$ atoms. For ${ }^{41} \mathrm{~K}$ the unbound halo is upshifted $\left(\left|\mu_{\mathrm{K}}\right|>\right.$ $\left.\left|\mu_{\mathrm{KRb}}\right|\right)$, whereas for ${ }^{87} \mathrm{Rb}$ it is downshifted $\left(\left|\mu_{\mathrm{Rb}}\right|<\left|\mu_{\mathrm{KRb}}\right|\right)$. By measuring the accelerations of the different components one could extract $\mu_{\mathrm{KRb}}$ and therefore the ratio of the atom numbers $N_{\mathrm{K}}^{d} / N_{\mathrm{Rb}}^{d}$ in the droplet. However, in free space, the acceleration is dominated by gravity (for the droplet the ratio between magnetic and gravitational forces is only 0.035 ).

\section{B. Waveguide dynamics}

To minimize the effect of gravity, we hold the atoms in a horizontal waveguide and let them evolve under the dominant effect of a magnetic field gradient along the $\hat{y}$ direction. In Fig. 2 we show in situ images of the two condensates as a function of the evolution time $t$ in the waveguide. In this configuration we are able to detect the bound states for a longer time, since the center-of-mass displacement is less than $300 \mu \mathrm{m}$ in $50 \mathrm{~ms}$. For $\delta g>0$ (top), both species expand as $t$ increases, while for $\delta g<0$ (bottom) we observe the formation of self-bound states. Also here, as well as in free space, a fraction of the atoms remains unbound and follows the same

${ }^{1}$ After $25 \mathrm{~ms}$ of TOF we estimate a variation of the Feshbach field of $0.17 \mathrm{G}$, resulting in an $a_{\mathrm{KRb}}$ variation of $2.9 a_{0}$.

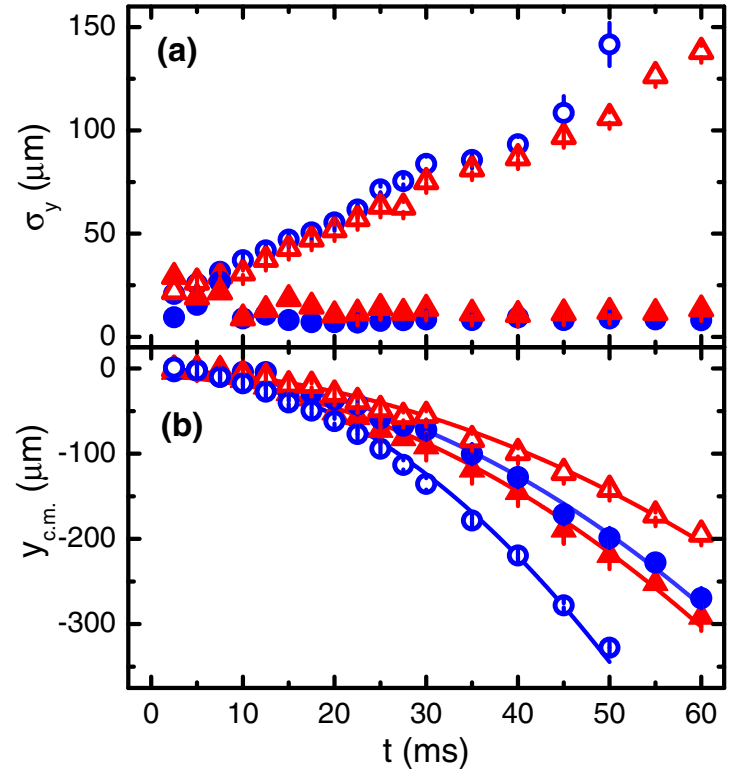

FIG. 3. Evolution of (a) the longitudinal size $\sigma_{y}$ and (b) the center-of-mass position $y_{\text {c.m. }}$ along the waveguide of ${ }^{41} \mathrm{~K}$ [blue open (closed) circles] and ${ }^{87} \mathrm{Rb}$ [red open (closed) triangles] clouds for $a_{\mathrm{KRb}}=(-11.4 \pm 1.4) a_{0}\left[(-82.5 \pm 1.6) a_{0}\right]$. Solid lines in (b) are fits with constant acceleration. The measured accelerations of the expanding clouds are $\alpha_{\mathrm{K}}=(0.276 \pm 0.025) \mathrm{m} / \mathrm{s}^{2}$ and $\alpha_{\mathrm{Rb}}=(0.104 \pm$ $0.010) \mathrm{m} / \mathrm{s}^{2}$ for $a_{\mathrm{KRb}}=(-11.4 \pm 1.4) a_{0}$, while for the bound components we find $\alpha_{d}=(0.152 \pm 0.017) \mathrm{m} / \mathrm{s}^{2}\left[\alpha_{d}=(0.151 \pm 0.022)\right.$ $\left.\mathrm{m} / \mathrm{s}^{2}\right]$ from images of ${ }^{41} \mathrm{~K}\left({ }^{87} \mathrm{Rb}\right)$. The error bars are the standard deviation of typically five independent measurements.

dynamics of the weakly attractive gas. In Fig. 3 we plot the size $\sigma_{y}$ and the center-of-mass position $y_{\text {c.m. }}$. along the longitudinal direction. For $\delta g<0$ we fit the atomic density distribution with two Gaussians to distinguish the bound and unbound components. For clarity, $\sigma_{y}$ and $y_{\text {c.m. }}$ are shown only for the bound components, since the unbound fraction behavior is very similar to the one observed for $\delta g>0$ (see Appendix C). The measured size for $\delta g<0$ (closed symbols) is constant and is comparable to the imaging resolution in the waveguide $(10 \mu \mathrm{m}){ }^{2}$ while for $\delta g>0$ both species expand (open symbols). In the waveguide, the center of mass moves under the combined effect of a magnetic force and a residual component of the gravity, since the guide is tilted $\theta \simeq 0.1^{\circ}-0.2^{\circ}$ with respect to the horizontal plane. Assuming a constant acceleration (see Appendix $\mathrm{C}$ ), we extract the ratio of the atom number of the two species in the bound state as

$$
\frac{N_{\mathrm{K}}^{d}}{N_{\mathrm{Rb}}^{d}}=\frac{m_{\mathrm{Rb}}\left(\alpha_{d}-\alpha_{\mathrm{Rb}}\right)}{m_{\mathrm{K}}\left(\alpha_{\mathrm{K}}-\alpha_{d}\right)},
$$

where $\alpha_{\mathrm{K}}$ and $\alpha_{\mathrm{Rb}}$ are the accelerations of the unbound ${ }^{41} \mathrm{~K}$ and ${ }^{87} \mathrm{Rb}$ clouds and $\alpha_{d}$ is the acceleration of the bound state. From $\alpha_{\mathrm{K}}$ and $\alpha_{\mathrm{Rb}}$ measured at $\delta g>0$ we find

\footnotetext{
${ }^{2}$ We use two different imaging systems: For measurements in free space (in the waveguide) the imaging beam propagates along the waveguide (crossed beam) and the optical resolution is $5 \mu \mathrm{m}$ (10 $\mu \mathrm{m})$.
} 


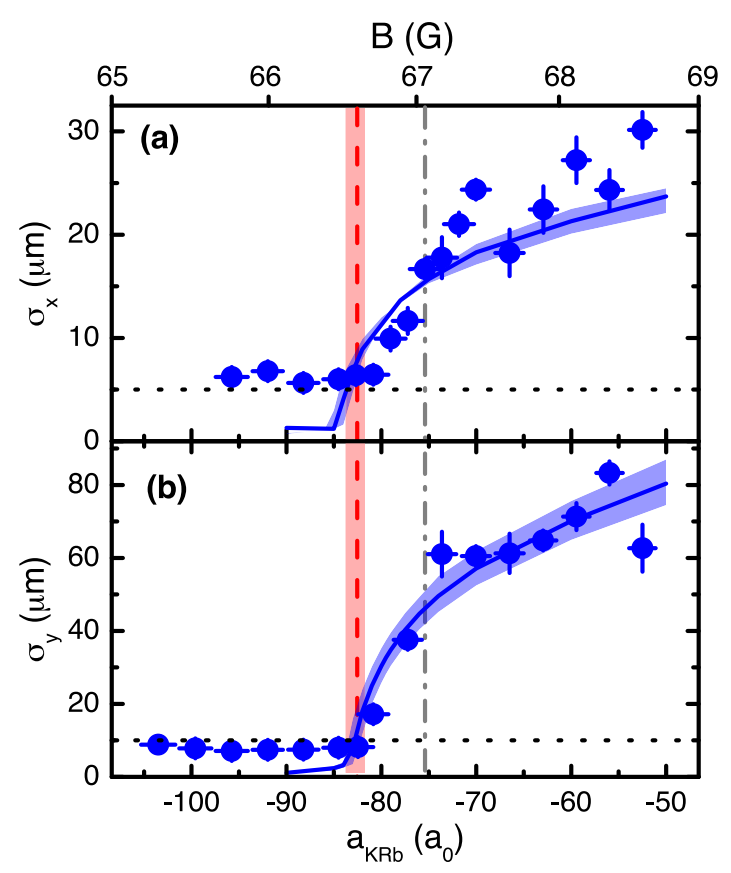

FIG. 4. (a) Size $\sigma_{x}$ of the ${ }^{41} \mathrm{~K}$ cloud expanding in free space as a function of the interspecies scattering length $a_{\mathrm{KRb}}$ after a TOF of $26.5 \mathrm{~ms}$ from the crossed trap. (b) Axial size $\sigma_{y}$ of the ${ }^{41} \mathrm{~K}$ cloud as a function of the interspecies scattering length $a_{\mathrm{KRb}}$ after expansion in the waveguide of $t=45 \mathrm{~ms}$. The dashed red vertical line corresponds to the critical $a_{\mathrm{KRb}}$ for the existence of a droplet in the homogeneous case with $N_{\mathrm{K}}+N_{\mathrm{Rb}}=33000$, while the dash-dotted gray vertical line corresponds to $\delta g=0$. The solid lines are the numerical predictions for $N_{\mathrm{K}}+N_{\mathrm{Rb}}=33000$, where we fix $N_{\mathrm{Rb}}=\sqrt{g_{\mathrm{K}} / g_{\mathrm{Rb}}} N_{\mathrm{K}}$. The colored areas indicate the systematic relative uncertainty of the experimental atom number of $30 \%$. We do not appreciate a substantial change of the average total atom number in the explored range of $a_{\mathrm{KRb}}$. The horizontal dotted lines show imaging resolutions of 5 and $10 \mu \mathrm{m}$ for the measurements in free space and in the waveguide, respectively. Vertical error bars are a standard deviation of typically five independent measurements.

$N_{\mathrm{K}}^{d} / N_{\mathrm{Rb}}^{d}=0.8(3)$, which is consistent with the predicted value of $\sqrt{g_{\mathrm{Rb}} / g_{\mathrm{K}}}=0.85$ [7]. We note that the center-of-mass motion allows a measurement of the atom number ratio which is not affected by the usual systematic uncertainty of $N$ measurements.

\section{Comparison with theory}

Both in free space and in the waveguide, we study the full range of attractive interactions. In Fig. 4 we plot the width $\sigma_{x}$ of ${ }^{41} \mathrm{~K}$ in free space after a TOF equal to $26.5 \mathrm{~ms}$ [Fig. 4(a)] and $\sigma_{y}$ after $t=45 \mathrm{~ms}$ of expansion in the waveguide [Fig. 4(b)]. In both cases we observe a transition from an expanding gas for $a_{\mathrm{KRb}} \gtrsim-82 a_{0}$ to a localized self-bound state for strong attractive interactions $\left(a_{\mathrm{KRb}} \lesssim-82 a_{0}\right)$ whose width is below the optical imaging resolution. In Fig. 4 we also show the MF threshold for collapse (dash-dotted gray line) and the critical scattering length to enter the droplet regime with our experimental atom number (dashed red line) [7]. Both lines are calculated for a homogeneous system. In the presence of the radial confinement a stable bound state may exist also in between the two vertical lines. This is due to the combined effect of the LHY term and the dispersion along the guide giving rise to the formation of "solitonic" solutions [18,31].

In order to compare the experimental results with theoretical predictions [7,21], we have performed numerical simulations by using two coupled generalized time-dependent GP equations including the LHY term, taking into account the actual experimental preparation (see Appendix B for details). In Fig. 4 the colored areas correspond to the numerical predictions including the experimental uncertainty on the atom number. Considering the finite imaging resolution, we find that the theory well reproduces the observed behavior. From the simulations in free space [Fig. 4(a)] we find that, in the strongly attractive regime, almost spherical droplets form [7]. As expected, the critical scattering length to enter the droplet regime depends on the atom number. We find that this threshold actually occurs at larger values of $\left|a_{\mathrm{KRb}}\right|$ with respect to the homogeneous case (see the dashed red line in Fig. 4). This is due to the additional kinetic energy cost associated with the droplet surface, whose effects are more important for small droplets, such as those formed in the experiment.

As for the waveguide dynamics, we observe expanding clouds in between the two vertical lines instead of the predicted solitonic bound states. We attribute this to a twofold effect: (i) We adjust the initial size of the mixture to match the droplet size (smaller than the one of the solitonic ground state) and (ii) the dynamics is triggered by a nonadiabatic process. However, droplets formed for very large attractive scattering lengths $\left(\left|a_{\mathrm{KRb}}\right| \gtrsim 95 a_{0}\right)$, as already observed in previous experiments [14], eventually decay into solitonic solutions due to the decrease of the atom number induced by three-body losses. In the waveguide, indeed, atomic losses do not affect the existence of self-bound states allowing their observation for a wider range of $a_{\mathrm{KRb}}$. In free space, instead, as confirmed by numerical simulations with three-body losses (Appendix B), we are limited to $\left|a_{\mathrm{KRb}}\right| \lesssim 95 a_{0}$. This corresponds to a broader range of $\delta g$ with respect to the case of ${ }^{39} \mathrm{~K}$ droplets, pushing the investigation far away from the MF collapse.

\section{CONCLUSION}

We have observed quantum droplets in a mixture of two atomic species experiencing different potentials, providing a clear picture of their formation and evolution both in free space and under radial confinement. This proves that the presence of identical traps for the two components is not essential to the droplets production. We have found evidence that the lifetime of our droplets is substantially longer than those in ${ }^{39} \mathrm{~K}$, as expected. The droplet density obtained by our numerical simulations results in a lifetime up to $400 \mathrm{~ms}$. This opens interesting perspectives for future studies of the droplets peculiar properties [7,21] and of their collective modes [31]. Indeed, the lower density of our sample allows for a larger ratio $\tau_{\text {life }} / \tau \sim n^{-1}$, with $\tau_{\text {life }}$ and $\tau$ being, respectively, the lifetime and the characteristic timescale of the droplet dynamics (Appendix B). Other directions could be the creation of droplets in reduced dimensionality [32-36], where 
quantum fluctuations become easily dominant, and the investigation of beyond LHY corrections [37] and other stabilization mechanisms [38,39]. Finally, the nucleation of vortexantivortex ring pairs [40-42] or more exotic scenarios, such as droplets clusters [43], could be studied with rotating droplets.

\section{ACKNOWLEDGMENTS}

We acknowledge inspiring discussions with M. Fattori. We thank M. Inguscio for support and A. Simoni for collisional calculations. This work was supported by the European Commission through FET Flagship on Quantum Technologies, Qombs Project (Grant No. 820419) and by Fondazione Cassa di Risparmio Firenze through project "SUPERACI-Superfluid Atomic Circuits." M.M. acknowledges support from the Spanish Ministry of Science, Innovation and Universities and the European Regional Development Fund FEDER through Grant No. PGC2018-101355-B-I00 (MCIU/AEI/FEDER, UE), and the Basque Government through Grant No. IT986-16.

\section{APPENDIX A: FESHBACH RESONANCES, MAGNETIC MOMENTS, AND THREE-BODY LOSSES}

In Fig. 5 we show the behavior of the interspecies scattering lengths $a_{\mathrm{KRb}}$ for ${ }^{41} \mathrm{~K}$ and ${ }^{87} \mathrm{Rb}$ in the $\left|F=1, m_{F}=1\right\rangle$ state as a function of the magnetic field $B$. Two Feshbach resonances around 40 and $80 \mathrm{G}$ can be seen. The values of $a_{\mathrm{KRb}}$ predicted by the two collisional models in Refs. [28,29]
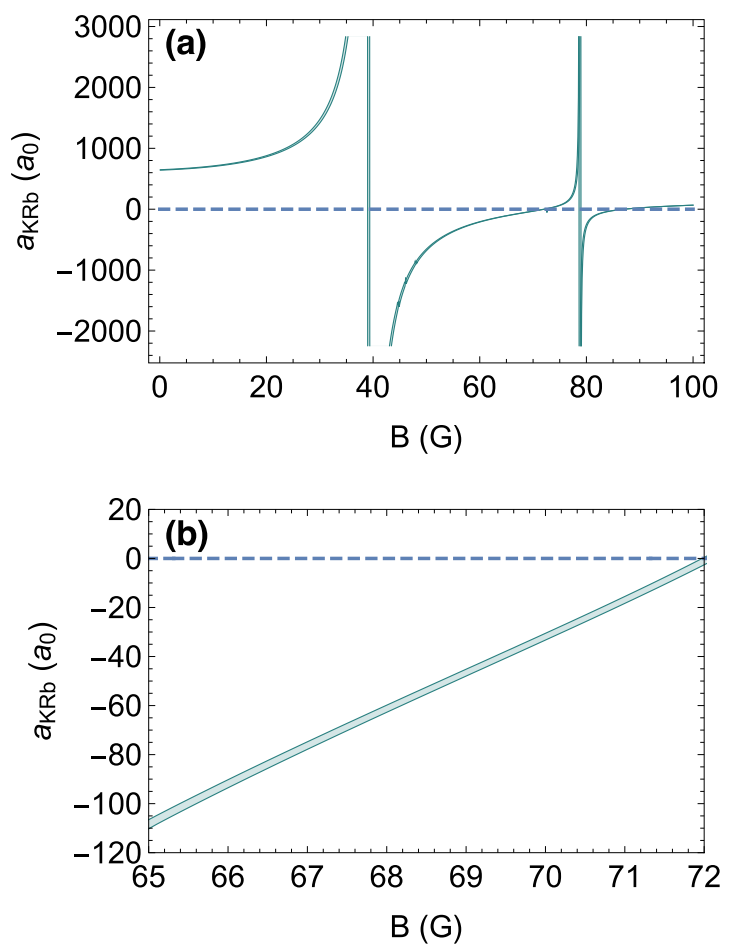

FIG. 5. (a) Calculated interspecies scattering length $a_{\mathrm{KRb}}$ for ${ }^{41} \mathrm{~K}$ and ${ }^{87} \mathrm{Rb}$ in the $\left|F=1, m_{F}=1\right\rangle$ state as a function of the magnetic field $B$. The predictions of both Refs. [28,29] are shown by the thickness of the curve. (b) Close-up of the range of magnetic field used in the experiment.

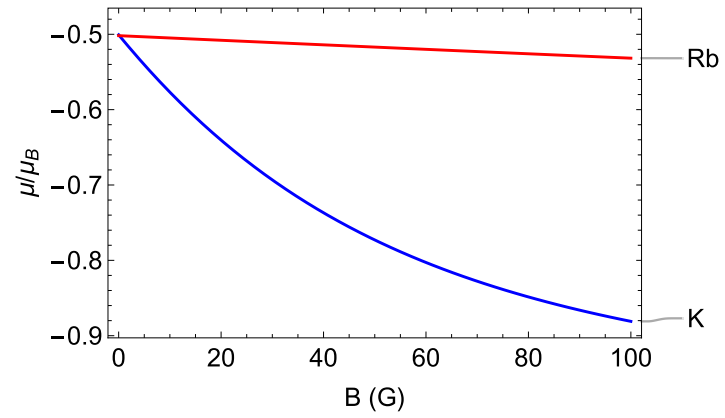

FIG. 6. Calculated magnetic moment $\mu$ (in units of the Bohr magneton $\mu_{\mathrm{B}}$ ) of the $\left|F=1, m_{F}=1\right\rangle$ state for both ${ }^{41} \mathrm{~K}$ and ${ }^{87} \mathrm{Rb}$ as a function of the magnetic field $B$.

differ slightly by a few $a_{0}$, as shown by the thickness of the curve in Fig. 5(b). All the values of $a_{\mathrm{KRb}}$ given in this work are the average of the two models, with uncertainty equal to the half-deviation that dominates over the one due to the magnetic field calibration.

In the experiment we produce the double condensate at $a_{\mathrm{KRb}} \simeq 0$ and then we tune the magnetic field in the range $65 \mathrm{G} \lesssim B \lesssim 69 \mathrm{G}$ to access the attractive interspecies interaction regime $\left(-100 a_{0} \lesssim a_{\mathrm{KRb}} \lesssim-50 a_{0}\right)$. In particular, we use two linear ramps: from $71 \mathrm{G}$ to $68.5 \mathrm{G}$ in $20 \mathrm{~ms}$ and then to the final value in the other $10 \mathrm{~ms}$.

At these magnetic fields, the Zeeman effect is no longer linear (especially for ${ }^{41} \mathrm{~K}$ ) and the magnetic moments $\mu_{\mathrm{K}}$ and $\mu_{\mathrm{Rb}}$ of the hyperfine state of ${ }^{87} \mathrm{Rb}$ and ${ }^{41} \mathrm{~K}$ in our mixture vary as shown in Fig. 6. In particular, in the range of $B$ used in the experiment the two magnetic moments are quite different, enabling a different magnetic control of the two atomic species, while their relative variations $\Delta \mu_{\mathrm{K}} / \mu_{\mathrm{K}}$ and $\Delta \mu_{\mathrm{Rb}} / \mu_{\mathrm{Rb}}$ are below $4 \%$ and therefore neglected. The difference between the two magnetic moments is exploited both to superimpose the two condensates by means of a magnetic gradient $b_{z}$ and to induce a different acceleration of the unbound and bound components.

As mentioned in Sec. III C, we include in our analysis a loss term due to inelastic three-body collisions. In general, the three-body loss coefficient $K_{3}$ depends on the scattering length, scaling as $a^{4}$ in the vicinity of a Feshbach resonance. In our case, we explored a very limited range of magnetic fields and scattering lengths $a_{\mathrm{KRb}}$; therefore, we measured the atom's lifetime to obtain the rate of inelastic three-body collisions at $a_{\mathrm{KRb}}=(-77.8 \pm 1.6) a_{0}$. We measured the lifetime of the ${ }^{41} \mathrm{~K}$ sample, since (i) the number of the minority species is more sensitive to losses and (ii) the dominant three-body losses are due to $\mathrm{K}-\mathrm{Rb}-\mathrm{Rb}$ collisions [44], yielding a purely exponential decay for ${ }^{41} \mathrm{~K}$ atoms. We measured a $1 / e$ lifetime $\tau_{\mathrm{K}}=122(11) \mathrm{ms}$ with an estimated ${ }^{87} \mathrm{Rb}$ peak density $n_{0, \mathrm{Rb}}=$ $(6 \pm 2) \times 10^{20} \mathrm{~m}^{-3}$. From $1 / \tau_{\mathrm{K}}=\left(K_{3} / 2 !\right) n_{0, \mathrm{Rb}}^{2} \xi$, with $\xi=$ $\int n_{\mathrm{Rb}}^{2} n_{\mathrm{K}} d V / n_{0, \mathrm{Rb}}^{2} N_{\mathrm{K}}$, we obtain $K_{3}=(7 \pm 4) \times 10^{-41} \mathrm{~m}^{6} / \mathrm{s}$ with an uncertainty due to that of $n_{0, \mathrm{Rb}}$. This must be compared to published values for larger scattering lengths: $K_{3} \simeq$ $10^{-38} \mathrm{~m}^{6} / \mathrm{s}$ at $a_{\mathrm{KRb}} \simeq-120 a_{0}[44]$ and $K_{3} \simeq 3 \times 10^{-39} \mathrm{~m}^{6} / \mathrm{s}$ at $a_{\mathrm{KRb}} \simeq-400 a_{0}$ [45]. 


\section{APPENDIX B: THEORETICAL ANALYSIS}

The GP energy functional for a Bose mixture, including both the mean-field term and the LHY correction accounting for quantum fluctuations in the local density approximation, reads $[7,21]$

$$
\begin{aligned}
E= & \sum_{i=1}^{2} \int d \boldsymbol{r}\left[\frac{\hbar^{2}}{2 m_{i}}\left|\nabla \psi_{i}(\boldsymbol{r})\right|^{2}+V_{i}(\boldsymbol{r}) n_{i}(\boldsymbol{r})\right] \\
& +\frac{1}{2} \sum_{i, j=1}^{2} g_{i j} \int d \boldsymbol{r} n_{i}(\boldsymbol{r}) n_{j}(\boldsymbol{r}) \\
& +\int d \boldsymbol{r} \mathcal{E}_{\mathrm{LHY}}\left(n_{1}(\boldsymbol{r}), n_{2}(\boldsymbol{r})\right),
\end{aligned}
$$

where $V_{i}(\boldsymbol{r})$ and $n_{i}(\boldsymbol{r})=\left|\psi_{i}(\boldsymbol{r})\right|^{2}$ represent the external potential and the density of each component $\left(i=1\right.$ for ${ }^{41} \mathrm{~K}$ and $i=2$ for ${ }^{87} \mathrm{Rb}$ ) and the LHY correction is [7]

$$
\begin{aligned}
\mathcal{E}_{\mathrm{LHY}} & =\frac{8}{15 \pi^{2}}\left(\frac{m_{1}}{\hbar^{2}}\right)^{3 / 2}\left(g_{11} n_{1}\right)^{5 / 2} f\left(\frac{m_{2}}{m_{1}}, \frac{g_{12}^{2}}{g_{11} g_{22}}, \frac{g_{22} n_{2}}{g_{11} n_{1}}\right) \\
& \equiv \kappa\left(g_{11} n_{1}\right)^{5 / 2} f(z, u, x) .
\end{aligned}
$$

Here $f(z, u, x)>0$ is a dimensionless function, whose expression for $z \neq 1$ and $u=1$ can be found in Ref. [21]. Following [7,21], we also consider this function at the mean-field collapse $u=1, f(z, 1, x)$. We note that the actual expression for $f$ can be fitted very accurately with the same functional form of the homonuclear case $\left(m_{1}=m_{2}\right)$ [46]

$$
f\left(\frac{87}{41}, 1, x\right) \simeq(1+\alpha x)^{\beta},
$$

where $\alpha$ and $\beta$ are fitting parameters that in general depend on the value of the mass ratio $m_{1} / m_{2}$. For the present case we find $\alpha \simeq 1.554$ and $\beta \simeq 2.506$, which are very close to the values of the approximated formula proposed in Ref. [46], which shows that only $\alpha$ is a function of the mass ratio $\alpha=$ $\left(m_{2} / m_{1}\right)^{3 / 5}=1.571$, whereas the value of $\beta$ is universal, $\beta=$ $5 / 2$.

Minimization of the action associated with Eq. (B1) leads to the generalized GP equations (Euler-Lagrange equations)

$$
i \hbar \frac{\partial \psi_{i}}{\partial t}=\left[-\frac{\hbar^{2}}{2 m_{i}} \nabla^{2}+V_{i}+\mu_{i}\left(n_{1}, n_{2}\right)\right] \psi_{i},
$$

where

$$
\mu_{i} \equiv \frac{\delta E}{\delta n_{i}}=g_{i i} n_{i}+g_{i j} n_{j}+\frac{\partial \mathcal{E}_{\mathrm{LHY}}}{\partial n_{i}} \quad(j \neq i)
$$

and

$$
\begin{gathered}
\frac{\partial \mathcal{E}_{\mathrm{LHY}}}{\partial n_{1}}=\kappa g_{11}\left(g_{11} n_{1}\right)^{3 / 2}\left(\frac{5}{2} f-x \frac{\partial f}{\partial x}\right), \\
\frac{\partial \mathcal{E}_{\mathrm{LHY}}}{\partial n_{2}}=\kappa g_{22}\left(g_{11} n_{1}\right)^{3 / 2} \frac{\partial f}{\partial x} .
\end{gathered}
$$

The above equations are solved by mapping the system (densities, wave functions, differential operators, etc.) on discrete equally spaced Cartesian grids. The differential operators are represented by a 13-point discretization. The stationary GP equation is solved by imaginary-time evolution. Dynamical equations have been solved by using Hamming's predictormodifier-corrector method, initiated by a fourth-order RungeKutta-Gill algorithm [47]. The effect of three-body losses on the real-time dynamics of the mixture is simulated by adding to the energy functional in Eq. (B1) a dissipative term $-(i / 2) \hbar K_{3} \int d \boldsymbol{r} n_{1}(\boldsymbol{r}, t) n_{2}(\boldsymbol{r}, t)^{2}$ (with $K_{3}=7 \times 10^{-41} \mathrm{~m}^{6} / \mathrm{s}$, see Appendix A) which accounts for the dominant recombination channel, i.e., K-Rb-Rb.

We performed two different series of simulations of the experiment for the TOF in free space and for the expansion in the waveguide. In the first case, the ground state of the mixture is produced in two concentric harmonic potentials (for the two condensates) approximating the actual crossed dipole trap. Then the potential is switched off abruptly and an evolution in free space is followed, taking into account three-body losses. For the simulation in the waveguide, we adopt a complete description of the potential experienced by the two species including the optical, magnetic, and gravitational potentials. This allows us to reproduce the effect of a residual magnetic field and a differential gravitational sag of the two components. The results reported in Fig. 4(b) are obtained, neglecting three-body losses after checking that their contribution is negligible in the range of parameters investigated. All results of the simulations shown in Fig. 4 have been performed with $N_{\mathrm{Rb}} / N_{\mathrm{K}}=\sqrt{g_{\mathrm{K}} / g_{\mathrm{Rb}}}$. Any unbalance from this ratio leads to an unbound expanding fraction which does not affect the bound fraction, but heavily affects computation time requiring a larger spatial grid. The simulations show the formation of self-bound states even when the trapping potentials of the two condensates are not identical and completely overlapped. A detailed investigation of this effect goes beyond the scope of the present work.

The equilibrium density of a droplet can be obtained with the condition $P=-\epsilon+\sum_{i}\left(\partial \epsilon / \partial n_{i}\right) n_{i}=0$, where $P$ is the pressure and $\epsilon$ is the energy density. For the heteronuclear case this condition gives

$$
\begin{aligned}
& n_{1}^{0}=\frac{25 \pi}{1024 a_{11}^{3}} \frac{\delta a^{2}}{a_{11} a_{22}} \frac{\left(m_{1}+m_{2}\right)^{2}}{4 m_{1} m_{2}}\left(1+\sqrt[10]{\frac{m_{2}}{m_{1}}} \sqrt{\frac{a_{22}}{a_{11}}}\right)^{-5}, \\
& n_{2}^{0}=n_{1}^{0} \sqrt{\frac{a_{11} m_{2}}{a_{22} m_{1}}} .
\end{aligned}
$$

Here $a_{11}, a_{22}$, and $a_{12}$ are the intra- and interspecies scattering lengths and $\delta a \equiv a_{12}-a_{12}^{c}$, with $a_{12}^{c}$ the mean-field threshold for collapse. From Eq. (B8) we estimate that, in our mixture, the homogeneous equilibrium density $n_{1}^{0}$ varies from $\sim 2 \times 10^{14}$ to $\sim 2 \times 10^{15}$ atoms $/ \mathrm{cm}^{3}$ for $-6 a_{0}<\delta a<$ $-20 a_{0}$, corresponding to the range for which we observe a droplet state [Fig. 4(a)]. Furthermore, for ${ }^{41} \mathrm{~K}$ and ${ }^{87} \mathrm{Rb}$, the terms containing the masses are $\sim 1$ and Eq. (B8) is not substantially different from the equation for the homonuclear case given in Ref. [7]:

$$
n_{1}^{0}=\frac{25 \pi}{1024 a_{11}^{3}} \frac{\delta a^{2}}{a_{11} a_{22}}\left(1+\sqrt{\frac{a_{22}}{a_{11}}}\right)^{-5} .
$$

From Eq. (B9) it is evident that upon increasing all scattering lengths by a factor $\gamma>1$, the densities decrease as $\gamma^{-3}$. To compare the equilibrium density of droplets formed either by the ${ }^{41} \mathrm{~K}-{ }^{87} \mathrm{Rb}$ mixture or with two spin states ${ }^{39} \mathrm{~K}$, we 
use Eqs. (B8) and (B9), respectively. In the first case $a_{11}$ and $a_{22}$ are larger than those of ${ }^{39} \mathrm{~K}$ (for which $a_{22}$ is tuned through a Feshbach resonance, while $a_{11}$ and $a_{12}$ are constant), resulting in a droplet density lower by approximately an order of magnitude at $\delta a$ of a few $-a_{0}$. As already mentioned in the main text, this also results in a longer lifetime $\left(\tau_{\text {life }} \sim n^{-2}\right)$ and a larger ratio $\tau_{\text {life }} / \tau \sim n^{-1}$, with $\tau$ the characteristic timescale of the droplet [7].

\section{APPENDIX C: WAVEGUIDE DYNAMICS}

\section{Equations of motion}

In this Appendix we derive the equations of motion for both atomic species confined in the waveguide. We start by considering the case in which they are unbound. The anticonfining force experienced by the $i$ th atomic species along the $\hat{y}$ axis is mainly determined by the magnetic potential $U_{y, i}$. This is given by the Feshbach field $B_{F}$ and the overlapping magnetic field gradient, which are produced by two different pairs of Helmholtz and anti-Helmholtz coils, respectively, along the $\hat{z}$ axis. Thus, we have

$$
U_{y, i}=-\left|\mu_{i}\right| \sqrt{B_{F}^{2}+\left(b_{y} y\right)^{2}},
$$

where $\mu_{i}$ is the magnetic moment and $b_{y}$ is the $\hat{y}$ component of the magnetic field gradient. For $\left|b_{y} y\right| \ll B_{F}$, which is fulfilled in our experimental conditions, $U_{y, i}$ can be approximated as

$$
U_{y, i} \simeq-\left|\mu_{i}\right| B_{F}\left[1+\frac{1}{2}\left(\frac{b_{y} y}{B_{F}}\right)^{2}\right],
$$

where we neglect the terms in $b_{y} y / B_{F}$ higher than second order. If we define $\omega_{i}^{2}=\left|\mu_{i}\right| /\left(m_{i} B_{F}\right) b_{y}^{2}$, with $m_{i}$ the atomic mass, the equation of motion is reduced to

$$
\ddot{y}_{i}-\omega_{i}^{2} y_{i}=0,
$$

where $y_{i}$ is the center-of-mass position of the atomic cloud and $\ddot{y}_{i}$ its acceleration. The solution of Eq. (C3) is

$$
y_{i}(t)=A_{1} \cosh \left(\omega_{i} t\right)+A_{2} \sinh \left(\omega_{i} t\right),
$$

where the constant factors $A_{1}$ and $A_{2}$ are determined by the initial conditions. For short times $\left(t \ll \min \left\{\omega_{i}^{-1}\right\}\right)$, Eq. (C4) can be approximated by its Taylor series expansion as

$$
y_{i}(t) \simeq y_{i}(0)\left[1+\frac{1}{2}\left(\omega_{i} t\right)^{2}\right]+\dot{y}_{i}(0) t,
$$

which corresponds to a uniformly accelerated motion, with constant acceleration $\alpha_{i} \equiv y_{i}(0) \omega_{i}^{2}$. Equation (C5) is a good approximation of the observed center-of-mass dynamics and indeed it has been used for fitting the experimental data discussed in the main text. Here $y_{i}(0)$ is the initial distance of the atomic cloud from the maximum of the magnetic potential and $\dot{y}_{i}(0)$ is the initial velocity. The value of $y_{i}(0)$ is not zero since the position of the atoms in the dipole trap is displaced from the symmetry axis of the magnetic field coils. We found that setting $\dot{y}_{i}(0)=0$ does not substantially affect the fitting results. Further we verified that, with $\dot{y}_{i}=0$, for evolution times up to $60 \mathrm{~ms}$ the error in $y_{i}$ made using Eq. (C5) instead of Eq. (C4) is less than $1 \%$.

We now treat the case of the bound state. Assuming that it is formed by $N_{i}^{d}$ atoms in each component $(i=1,2)$, then its

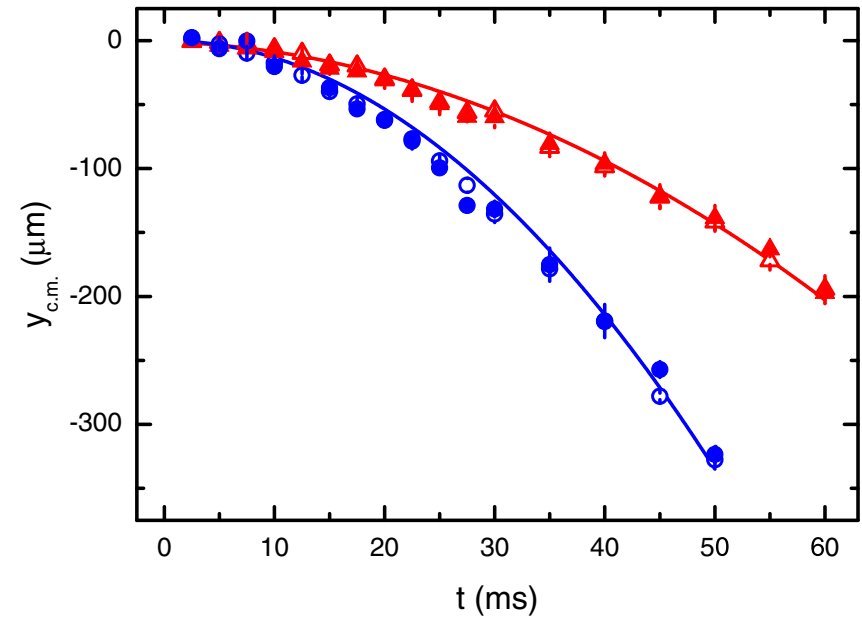

FIG. 7. Evolution of the center-of-mass position $y_{\text {c.m. }}$ along the waveguide for $a_{\mathrm{KRb}}=(-11.4 \pm 1.4) a_{0}$ of ${ }^{41} \mathrm{~K}$ (blue open circles) and ${ }^{87} \mathrm{Rb}$ (red open triangles) and for the expanding fractions at $a_{\mathrm{KRb}}=(-82.5 \pm 1.6) a_{0}$ of ${ }^{41} \mathrm{~K}$ (blue closed circles) and ${ }^{87} \mathrm{Rb}$ (red closed triangles). Solid lines are fit with constant acceleration for $a_{\mathrm{KRb}}=(-82.5 \pm 1.6) a_{0}$. The fit results are $\alpha_{\mathrm{K}}=(-0.27 \pm 0.07)$ $\mathrm{m} / \mathrm{s}^{2}$ and $\alpha_{\mathrm{Rb}}=(-0.104 \pm 0.013) \mathrm{m} / \mathrm{s}^{2}$.

equation of motion is given by

$$
\ddot{y}_{d} \sum_{i=1}^{2} N_{i}^{d} m_{i}=\sum_{i=1}^{2} F_{i},
$$

where $y_{d}$ is the center of mass of the droplet and $F_{i}$ is the total (constant) force acting on each component. This force can also be expressed in terms of the center-of-mass acceleration $\alpha_{i}$ that the $i$ th component would have in the absence of the other species, namely, $F_{i}=N_{i}^{d} m_{i} \alpha_{i}$. Then Eq. (C6) can be rewritten as

$$
\ddot{y}_{d} \sum_{i} N_{i}^{d} m_{i}=\sum_{i} N_{i}^{d} m_{i} \alpha_{i}
$$

from which it is straightforward to get Eq. (2).

\section{Experimental fit of the unbound-state dynamics}

In the droplet regime, we generally observe a portion of atoms left over the bound state. We ascribe this effect to the presence of a thermal fraction in both atomic species and an excess of ${ }^{87} \mathrm{Rb}$ atoms with respect to the droplet atom ratio. Thus, to distinguish the bound and unbound parts, we fit the atomic density distribution with a two-component Gaussian function. In Fig. 4 we have shown only the results obtained for the bound portion. Here we report also the evolution of the center-of-mass position $y_{\text {c.m. }}$. of the unbound fraction for $\delta g<0$, finding the same behavior of the weakly attractive mixture (Fig. 7).

Following the same strategy described in the main text, from Eq. (2), where now $\alpha_{\mathrm{K}}$ and $\alpha_{\mathrm{Rb}}$ are the accelerations of the unbound ${ }^{41} \mathrm{~K}$ and ${ }^{87} \mathrm{Rb}$ components at $\delta g<0$, we obtain the atom number ratio of the two species in the bound state, $N_{\mathrm{K}}^{d} / N_{\mathrm{Rb}}^{d}=0.8 \pm 0.7$, again consistent with the expected value [7]. 
[1] T. D. Lee, K. Huang, and C. N. Yang, Phys. Rev. 106, 1135 (1957).

[2] A. Altmeyer, S. Riedl, C. Kohstall, M. J. Wright, R. Geursen, M. Bartenstein, C. Chin, J. H. Denschlag, and R. Grimm, Phys. Rev. Lett. 98, 040401 (2007).

[3] Y.-i. Shin, A. Schirotzek, C. H. Schunck, and W. Ketterle, Phys. Rev. Lett. 101, 070404 (2008).

[4] S. B. Papp, J. M. Pino, R. J. Wild, S. Ronen, C. E. Wieman, D. S. Jin, and E. A. Cornell, Phys. Rev. Lett. 101, 135301 (2008).

[5] N. Navon, S. Nascimbène, F. Chevy, and C. Salomon, Science 328, 729 (2010).

[6] N. Navon, S. Piatecki, K. Günter, B. Rem, T. C. Nguyen, F. Chevy, W. Krauth, and C. Salomon, Phys. Rev. Lett. 107, 135301 (2011).

[7] D. S. Petrov, Phys. Rev. Lett. 115, 155302 (2015).

[8] H. Kadau, M. Schmitt, M. Wenzel, C. Wink, T. Maier, I. FerrierBarbut, and T. Pfau, Nature (London) 530, 194 (2016).

[9] I. Ferrier-Barbut, H. Kadau, M. Schmitt, M. Wenzel, and T. Pfau, Phys. Rev. Lett. 116, 215301 (2016).

[10] M. Schmitt, M. Wenzel, F. Böttcher, I. Ferrier-Barbut, and T. Pfau, Nature (London) 539, 259 (2016).

[11] L. Chomaz, S. Baier, D. Petter, M. J. Mark, F. Wächtler, L. Santos, and F. Ferlaino, Phys. Rev. X 6, 041039 (2016).

[12] I. Ferrier-Barbut, M. Schmitt, M. Wenzel, H. Kadau, and T. Pfau, J. Phys. B 49, 214004 (2016).

[13] M. Wenzel, F. Böttcher, T. Langen, I. Ferrier-Barbut, and T. Pfau, Phys. Rev. A 96, 053630 (2017).

[14] P. Cheiney, C. R. Cabrera, J. Sanz, B. Naylor, L. Tanzi, and L. Tarruell, Phys. Rev. Lett. 120, 135301 (2018).

[15] C. R. Cabrera, L. Tanzi, J. Sanz, B. Naylor, P. Thomas, P. Cheiney, and L. Tarruell, Science 359, 301 (2018).

[16] G. Semeghini, G. Ferioli, L. Masi, C. Mazzinghi, L. Wolswijk, F. Minardi, M. Modugno, G. Modugno, M. Inguscio, and M. Fattori, Phys. Rev. Lett. 120, 235301 (2018).

[17] G. Ferioli, G. Semeghini, L. Masi, G. Giusti, G. Modugno, M. Inguscio, A. Gallemí, A. Recati, and M. Fattori, Phys. Rev. Lett. 122, 090401 (2019)

[18] V. E. Zakharov and A. B. Shabat, Sov. Phys. JETP 34, 62 (1972).

[19] E. P. Gross, J. Math. Phys. 4, 195 (1963).

[20] L. P. Pitaevskii, Sov. Phys. JETP 13, 451 (1961).

[21] F. Ancilotto, M. Barranco, M. Guilleumas, and M. Pi, Phys. Rev. A 98, 053623 (2018).

[22] A. Burchianti, C. D’Errico, S. Rosi, A. Simoni, M. Modugno, C. Fort, and F. Minardi, Phys. Rev. A 98, 063616 (2018).

[23] G. Thalhammer, G. Barontini, L. De Sarlo, J. Catani, F. Minardi, and M. Inguscio, Phys. Rev. Lett. 100, 210402 (2008).
[24] A. H. Hansen, A. Y. Khramov, W. H. Dowd, A. O. Jamison, B. Plotkin-Swing, R. J. Roy, and S. Gupta, Phys. Rev. A 87, 013615 (2013).

[25] R. S. Lous, I. Fritsche, M. Jag, B. Huang, and R. Grimm, Phys. Rev. A 95, 053627 (2017).

[26] C. D’Errico, M. Zaccanti, M. Fattori, G. Roati, M. Inguscio, G. Modugno, and A. Simoni, New J. Phys. 9, 223 (2007).

[27] A. Marte, T. Volz, J. Schuster, S. Dürr, G. Rempe, E. G. M. van Kempen, and B. J. Verhaar, Phys. Rev. Lett. 89, 283202 (2002).

[28] A. Simoni, M. Zaccanti, C. D’Errico, M. Fattori, G. Roati, M. Inguscio, and G. Modugno, Phys. Rev. A 77, 052705 (2008).

[29] G. Thalhammer, G. Barontini, J. Catani, F. Rabatti, C. Weber, A. Simoni, F. Minardi, and M. Inguscio, New J. Phys. 11, 055044 (2009).

[30] F. Riboli and M. Modugno, Phys. Rev. A 65, 063614 (2002).

[31] A. Cappellaro, T. Macrì, and L. Salasnich, Phys. Rev. A 97, 053623 (2018).

[32] D. S. Petrov and G. E. Astrakharchik, Phys. Rev. Lett. 117, 100401 (2016).

[33] D. Edler, C. Mishra, F. Wächtler, R. Nath, S. Sinha, and L. Santos, Phys. Rev. Lett. 119, 050403 (2017).

[34] G. E. Astrakharchik and B. A. Malomed, Phys. Rev. A 98, 013631 (2018).

[35] P. Zin, M. Pylak, T. Wasak, M. Gajda, and Z. Idziaszek, Phys. Rev. A 98, 051603(R) (2018).

[36] L. Parisi, G. E. Astrakharchik, and S. Giorgini, Phys. Rev. Lett. 122, 105302 (2019).

[37] V. Cikojević, L. V. Markić, G. E. Astrakharchik, and J. Boronat, Phys. Rev. A 99, 023618 (2019).

[38] S. K. Adhikari, Phys. Rev. A 95, 023606 (2017).

[39] S. Gautam and S. K. Adhikari, J. Phys. B 52, 055302 (2019).

[40] Y. V. Kartashov, B. A. Malomed, L. Tarruell, and L. Torner, Phys. Rev. A 98, 013612 (2018).

[41] F. Ancilotto, M. Barranco, and M. Pi, Phys. Rev. B 97, 184515 (2018).

[42] J. M. Escartín, F. Ancilotto, M. Barranco, and M. Pi, Phys. Rev. B 99, 140505(R) (2019).

[43] Y. V. Kartashov, B. A. Malomed, and L. Torner, Phys. Rev. Lett. 122, 193902 (2019).

[44] L. J. Wacker, N. B. Jorgensen, D. Birkmose, N. Winter, M. Mikkelsen, J. Sherson, N. Zinner, and J. J. Arlt, Phys. Rev. Lett. 117, 163201 (2016).

[45] K. Kato, Y. Wang, J. Kobayashi, P. S. Julienne, and S. Inouye, Phys. Rev. Lett. 118, 163401 (2017).

[46] F. Minardi, F. Ancilotto, A. Burchianti, C. D’Errico, C. Fort, and M. Modugno, arXiv:1908.00804.

[47] A. Ralston and H. S. Wilf, Mathematical Methods for Digital Computers (Wiley, New York, 1960). 